\title{
Investigating Anticancer Potency of In Vitro Propagated Endemic Thymus Cilicicus Boiss. \& Bal. Extract on Human Lung, Breast, and Prostate Cancer Cell Lines
}

\section{Naeem Abdul Ghafoor ( $\sim$ merzanaeem007@gmail.com )}

Mugla Sitki Kocman University: Mugla Sitki Kocman Universitesi https://orcid.org/0000-0002-42007679

\section{Selin Galatali}

Mugla Sitki Kocman University: Mugla Sitki Kocman Universitesi

\section{Sevil Yeniocak}

Mugla Sitki Kocman University: Mugla Sitki Kocman Universitesi

\section{Ergun Kaya}

Mugla Sitki Kocman University: Mugla Sitki Kocman Universitesi

\section{Nurdan Sarac}

Mugla Sitki Kocman University: Mugla Sitki Kocman Universitesi

\section{Aysel Ugur}

Gazi University: Gazi Universitesi

\section{Research Article}

Keywords: Cancer, Thymus cilicicus, lung cancer, breast cancer, prostate cancer, anticancer agents, molecular docking

Posted Date: January 18th, 2022

DOI: https://doi.org/10.21203/rs.3.rs-1206301/v1

License: (c) (i) This work is licensed under a Creative Commons Attribution 4.0 International License. Read Full License 


\title{
1 Investigating anticancer potency of in vitro propagated endemic Thymus 2 cilicicus Boiss. \& Bal. extract on human lung, breast, and prostate cancer cell 3 lines
}

\author{
4 Naeem Abdul Ghafoor ${ }^{1 *}$, Selin Galatali ${ }^{1}$, Sevil Yeniocak $^{1}$, Ergun Kaya $^{1}$, Nurdan Sarac ${ }^{2 *}$, Aysel Ugur $^{3}$ \\ 5 'Department of Molecular Biology and Genetics, Faculty of Science, Mugla Sitki Kocman University, \\ 6 Mugla, Turkey. \\ $7 \quad{ }^{2}$ Department of Biology, Faculty of Science, Mugla Sitki Kocman University, Mugla, Turkey \\ $8{ }^{3}$ Section of Medical Microbiology, Department of Basic Sciences, Faculty of Dentistry, Gazi University, \\ 9 Ankara, Turkey
}

*Corresponding authors: merzanaeem007@gmail.com, nsarac@ mu.edu.tr

\section{Abstract}

Cancer is the leading cause of death in the world, accounting for nearly 10 million deaths in 2020 alone and surpassing all other categories by a large margin. Several different strategies have been and are still being deployed to combat different types of cancers and among the common clinical approaches are the use of synthetic and natural compounds as anticancer agents within chemotherapy regimens. Thymus cilicicus Boiss. \& Bal is a spice endemic to Turkey, the Northern Aegean Islands, Lebanon, and Syria, while several species of the Thymus genus are known to exhibit different clinically valuable properties, the research on T. cilicicus is rather scarce, therefore, in this study, the wound healing properties of in vitro propagated $T$. cilicicus ethanolic extracts were investigated on murine fibroblast (NIH-3T3), and its anticancer potency was investigated on the human alveolar basal epithelial adenocarcinoma (A549), human breast adenocarcinoma (MDA-MB-213), and human prostate cancer (DU-145) cell lines via colorimetric MTT assays. The wound healing property assessments didn't lead to any significant results, however, T. cilicicus yielded selective and promising anticancer potency on the A549 cell line. Furthermore, molecular docking analyses were on the proteins of 9 genes confirmed to be upregulated in both 3D and 4D A549 cultures against 48 compounds found in the essential oils of $T$. cilicicus were performed and yielded results acquiescent with previous findings in the scientific literature. This study provides in vitro evidence to the selective anticancer activity of T. cilicicus extracts on A549 cells enhanced with computational evidence on the molecular mechanism involved in this selective activity. This study serves as a precursor for further in vivo and clinical research on the constituents of $T$. cilicicus as potent anticancer agents and their potential use in cancer therapies.

Keywords: Cancer; Thymus cilicicus; lung cancer; breast cancer ; prostate cancer; anticancer agents; molecular docking

\section{Introduction}

Cancer is the leading cause of death in the world, accounting for nearly 10 million deaths in 2020 alone, surpassing all other categories by a large margin (WHO 2021). While 30-50\% of cancer cases could be prevented by avoiding the risk factors and implementing prevention strategies, the remaining cases require some form of clinical assistance. The oldest record for a cancer case has been reported from breast 
cancer in ancient Egypt around 1500 BC, cancer develops in normal cells as they starts proliferating uncontrollably, i.e. when normal healthy cells grow and proliferate without any limitation or restrictions (Sudhakar 2009). DNA damage resulting in tens of thousands of individual molecular lesions per cell per day constantly occur in healthy cells under their regular metabolic activity or when exposed to certain environmental factors, however, under normal circumstances, DNA repair mechanisms recruit several cellular elements through which it identifies and repairs the damage to the DNA molecule, nevertheless, every once in a while depending on the genetic composition of the cell and the environmental factors it's exposed to, certain damage to specific genes aren't properly rectified and hence result in the formation of tumor cells (Lodish et al. 2008). Five major mechanisms involved in this rectification process are, the base excision repair, nucleotide excision repair, mismatch repair, repair by homologous recombination, and repair by non-homologous end joining, different combinations of these pathways are known to be active during different stages of the cell cycle so as to maintain the integrity of the cell's genome and repair any damage, failure of these molecular mechanisms lead to survival and proliferation of the cells with damaged DNA which reflects as tumors (Chatterjee and Walker 2017).

The usage of natural compounds from plants, animals, and microorganisms by humans to treat different medical conditions dates long before recorded history, some paleoanthropological studies done in the Middle East concluded that Neanderthals might have been aware of the medicinal properties of several medicinal plants more than 60 thousand years ago (Solecki 1975). The oldest record mentioning the usage of medicinal plants for their activities comes from ancient Mesopotamia, around $2600 \mathrm{BC}$, which is not a surprise given that they're a civilization known for keeping written records. The records describe around 1 thousand plants (and their compounds) along with their medicinal application, some of which are still in use nowadays (Newman, Cragg, and Snader 2000). While human ancestors used plant products to relieve pain or improve wound healing without any knowledge of their action mechanisms, recent developments in molecular and cellular biology provide the opportunities to investigate the mechanism of action for these natural products to use them more efficiently and assess their side effects and risks (Schenone et al. 2013). The genus Thymus is a member of the Lamiaceae family and constitutes of 214 species spread across North Africa, temperate Asia zone, and Europe, several members of this genus have been widely used in food, cosmetics, perfumery, and pharmaceutical industries, they're also known to be widely used in traditional medicine to treat digestive disorders, cough, diarrhea, headache, cold, bronchitis, renal stone, asthma, and many other diseases, their essential oils contain several phenolic derivatives, terpenes, and esters, most of which has been extensively researched (Salehi et al. 2019; Cornara et al. 2009; Alarcón et al. 2015; Rowshan, Bahmanzadegan, and Saharkhiz 2013; X. Li et al. 2019). Several studies have also revealed that extracts from several species of the Thymus genus possess antimicrobial, antioxidant, antitumor, antiinflammatory, analgesic, and other medically valuable properties (X. Li et al. 2019; Nabavi et al. 2015; Sarac and Ugur 2008).

Previous in vivo and in vitro studies that investigated the extracts from $T$. quinquecostatus, $T$. vulgaris, T. carnosus, T. kotschyanus, T. mastichina, and T. citriodorus also concluded their anticancer potentials (Martins-Gomes et al. 2019; S. Wu et al. 2013; Martins-Gomes et al. 2018; Sun et al. 2005; Heidari et al. 2018; Doosti, Ahmadi, and Fasihi-Ramandi 2018; Gordo et al. 2012; Abaza et al. 2015). Further in-depth investigations into thymol (2-isopropyl-5-methylphenol, 36.0-55.0\% of essential oils constituent, found in most of the Thymus species) had designated that it showed anticancer efficacy against human liver cancer (Bel-7402), breast cancer (MCF-7), acute promyelocytic leukemia (HL-60), bladder cancer (T24, SW780, and J82), immortalized urothelial (SV-HUC-1), colon adenocarcinoma (Caco-2), ovarian cancer (SKOV-3) and colon cancer (HCT116 and LoVo) cell lines (QingHua, YinZhi, and FengXiang 2010; Seresht et al. 2019; Deb et al. 2011; Y. Li et al. 2017; Llana-Ruiz-Cabello et al. 2014; Zeng et al. 2020; 
Elbe et al. 2020). Two other extracts, oleanolic acid and ursolic acid isolated from T. mastichina L. have also been reported to exert improved synergistic anticancer activity on colon cancer (HCT-116) cell lines (Gordo et al. 2012). Carvacrol, another monoterpene phenol found in the essential oils several Thymus species are known to exert anticancer activity on human alveolar basal epithelial adenocarcinoma (A549), hepatocellular carcinoma (HepG-2), breast cancer (MCF-7), and ovarian cancer (SKOV-3) cell lines (Koparal and Zeytinoglu 2003; Elbe et al. 2020; Esmaeili-Mahani, Falahi, and Yaghoobi 2014; Patel, Shah, and Bavadekar 2012; Yin et al. 2012). Rosmarinic acid has also been reported to possess the ability to inhibit the proliferation of human colorectal adenocarcinoma (HT-29) cell lines with synergistic activity on some other cancerous cell lines (Erenler et al. 2016). Similarly, apigenin, a dietary flavonoid found in the former plants is known for its cell-cycle arrest and apoptosis induction activity in different tumor types such breast, cervical, colon, lung, liver, prostate, pancreatic, and stomach (X. Li et al. 2019; Imran et al. 2020; Erenler et al. 2016).

Thymus cilicicus Boiss. \& Bal is a member of the Thymus genus, it is endemic to Turkey, the Northern Aegean Islands, Lebanon, and Syria and is sold as a spice and herbal tea only in the Çaml1 (Chamli) village of Marmaris province in Mugla (Turkey), its locally known as lemon thyme, fish thyme, and cheese thyme and commonly used for stomachache relief, it's also reported in the literature for its use as a tranquilizer and for toothache relief. They can be consumed fresh at any time of the year or harvested when they bloom and distilled in oil or dried for later use (Baser 2002; Gürdal and Kültür 2013; Everest and Ozturk 2005). In a study conducted by Sarac and Ugur (2008), essential oils of T. cilicicus collected from various regions of Mugla province were obtained by hydrodistillation and their antimicrobial activity against some microorganisms was investigated, including multi-antibiotic-resistant bacteria, and grampositive bacterias, and yielded promising results.

In this study, the aerial shoots of in vitro propagated T. cilicicus. were isolated and dried, ethanol extraction was performed on the dried leaves and the extract was analyzed for their wound healing property on murine fibroblast (NIH-3T3), and their antiproliferative activity on NIH-3T3, human alveolar basal epithelial adenocarcinoma (A549), human breast adenocarcinoma (MDA-MB-231), and human prostate cancer (DU-145) cell lines. Computational analyses such as molecular docking was utilized to elucidate the molecular mechanism underlying the potent antiproliferation activity on selected cell lines.

\section{Materials \& Methods}

\subsection{Preparing the plant material}

In vitro cultures of T. cilicicus Boiss. \& Bal. was obtained by following the optimum propagation protocol reported by (Kaya et al. 2021). The aerial shoots of the plant were isolated and left to dry on top of filter papers inside a dry air ventilating incubator at $30^{\circ} \mathrm{C}$ for 24 hours, after 24 hours, the dry leaves were weighed and transferred into a mortar. Liquid nitrogen was added until all the dry leaves were immersed and they were milled by grinding until the liquid nitrogen evaporated and fine powders were obtained. The dry powder was weighed and taken into a falcon into which $10 \mathrm{~mL}$ ethanol (99.9\% pure laboratory-grade) was added, the falcon was vortexed and placed into an ultrasonic bath for 1 hour. After 1 hour, the falcon was vortexed again and the content was filtered into a clear beaker via a filter paper and left in a fume hood overnight to allow the ethanol to evaporate. The dry extract was dissolved in Dimethyl sulfoxide (DMSO, $99.98 \%$ pure laboratory-grade) at a final concentration of $0.2 \mathrm{~g} / \mathrm{mL}$.

\subsection{Cell culturing and extract preparation}


A fresh batch of NIH-3T3 (RRID:CVCL_0594) cell line was thawed from a -86 ${ }^{\circ} \mathrm{C}$ stock and seeded into a sterile T75 flask with $15 \mathrm{~mL}$ fresh high glucose DMEM (Sigma Dulbecco's Modified Eagle Medium, supplemented with $10 \%$ fetal bovine serum, and $1 \% 10^{4} \mathrm{U} / \mathrm{mL}$ streptomycin, $10^{5} \mu \mathrm{g} / \mathrm{mL}$ penicillin, and $25 \mu \mathrm{g} / \mathrm{mL}$ amphotericin B mix) and left to incubate in a humidified atmosphere with $5 \%$ $\mathrm{CO}_{2}$ at $37{ }^{\circ} \mathrm{C}$ until it reached $80 \%$ confluency (changing the medium every 24 hours). After reaching $80 \%$ confluency, the cells were harvested and seeded into a sterile 96-well cell microplate at a concentration of $10^{4}$ cells per well per $200 \mu \mathrm{L}$. The same procedure was followed to prepare the cell microplates for the A549 (RRID:CVCL_0023), MDA-MB-231 (RRID:CVCL_0062), and DU-145 (RRID:CVCL_0105) cell lines. Each cell plate was incubated in a humidified atmosphere with $5 \% \mathrm{CO}_{2}$ at $37{ }^{\circ} \mathrm{C}$ for 24 hours.

Before applying the $T$. cilicicus extracts to the cell lines, the stock concentration of the extract $(0.2$ $\mathrm{g} / \mathrm{mL}$ ) was diluted 1/40-fold in the medium (DMEM) to obtain a working solution with a concentration of $2 \mathrm{mg} / \mathrm{mL}$, further dilutions (with DMEM) were performed to obtain aliquots with concentrations ranging between 1000 to $100 \mu \mathrm{g} / \mathrm{mL}$. The same dilution ratio was used to dilute DMSO (1/40-fold in DMEM) which was further diluted to obtain aliquots with concentrations ranging between 1000 to $100 \mu \mathrm{g} / \mathrm{mL}$ (normalized control group). 1/40-fold dilution of the initial plant material and DMSO was performed to standardize the toxic effects of DMSO on the cell lines (standardizing the toxicity of the solvents).

\subsection{Colorimetric MTT assay}

After incubating the 96-well cell microplates seeded with NIH-3T3, A549, MDA-MB-231, and DU-145 cell lines for 24 hours, their mediums was discarded and $200 \mu \mathrm{L}$ of each of the extract aliquots at concentrations of $1000,900,800,700,600,500,400$, and $300 \mu \mathrm{g} / \mathrm{mL}$ was added to 6 wells in each row (i.e. sextuple replicas) and their DMSO controls were added to the remaining 6 wells of the same row at the same concentration (i.e., each row contained 6 wells with extract aliquots at a particular concentration and 6 wells with the DMSO aliquots at the same concentration), each 96-well cell microplate was incubated in a humidified atmosphere with $5 \% \mathrm{CO}_{2}$ at $37{ }^{\circ} \mathrm{C}$ for 24 hours. After 24 hours, the content of each plate was discarded and the wells in each plate were rinsed with $100 \mu \mathrm{L}$ D-PBS (Dulbecco's phosphate-buffered saline, Pan Biotech, Turkey), and $200 \mu \mathrm{L}$ fresh DMEM and $20 \mu \mathrm{L}$ MTT reagent (3-(4,5-dimethylthiazol2-yl)-2,5-diphenyltetrazolium bromide prepared in D-PBS at a concentration of $5 \mathrm{mg} / \mathrm{mL}$ ) was added to each well. The plates were left in a humidified atmosphere with $5 \% \mathrm{CO}_{2}$ at $37{ }^{\circ} \mathrm{C}$ for 3 hours after which their content was discarded (without disturbing the formazan crystals at the bottom of the wells) and 100 $\mu \mathrm{L}$ DMSO was added to each well of the cell plates and they were incubated at $37^{\circ} \mathrm{C}$ in a shaking incubator at $120 \mathrm{rpm}$ for 20 minutes to dissolve the formazan crystals formed by MTT. After the incubation, the absorbance of each microplate was immediately measured at $540 \mathrm{~nm}$ in a spectrophotometer.

To calculate the viability of the cell, the absorbance of the wells incubated with plant extract was divided by the average absorbance of their corresponding DMSO control and converted into a percentage by multiplying by 100 (cell viability percentage). The inhibition percentages were calculated via $E q$. 1, where $A_{540, X}$ is the absorbance of a well incubated with X concentration of the plant extract at $540 \mathrm{~nm}$, and $A_{540, \Delta C X}$ is the average absorbance of the control group at the same (X) concentration. A logarithmic plot of the concentration against their respective inhibition percentages was plotted and the $\mathrm{IC}_{50}$ (i.e., the concentration required for $50 \%$ proliferation inhibition) was calculated from the equation of the line by substituting 50 for y. SciPy (RRID:SCR_008058) was used to fit the data and their plots were generated via MatPlotLib (RRID:SCR_008624) and seaborn (RRID:SCR_018132) libraries in Python enviroment (Hunter 2007; Waskom 2021; Virtanen et al. 2020).

$$
\text { Inhibtion } \%=100-\text { Cell viability (\%) }
$$




$$
\text { Cell viability }(\%)=\left(\frac{A_{540, X}}{A_{540, \Delta C X}} \times 100\right)
$$

\subsection{Scratch wound assay}

172

173

174

175

176

177

178

179

180

181

182

183

A portion of the freshly thawed NIH-3T3 cells was seeded into 6 sterile Petri dishes $(2 \mathrm{r}=60 \mathrm{~mm})$ at a concentration of $1.25 \times 10^{5}$ cells per Petri dish and was incubated in a humidified atmosphere with 5\% $\mathrm{CO}_{2}$ at $37{ }^{\circ} \mathrm{C}$ until they were completely coated with cells (confluency $>90 \%, \approx 24$ hours), after the incubation period, their mediums were discarded, and scratch wound assay (artificial wound drawn across the Petri dish) was performed on each of the 6 Petri dishes. The Petri dishes were rinsed in D-PBS and 2.5 $\mathrm{mL}$ of DMEM containing 100, 200, 300, and $400 \mu \mathrm{g} / \mu \mathrm{L}$ plant extract (1/40 diluted in DMEM as explained above, doses selected were below the IC50 obtained from the MTT assay in the previous step) was applied to the first 4 Petri dishes, $2.5 \mathrm{~mL}$ 1/40-fold diluted DMSO was applied to the $5^{\text {th }}$ Petri dish (normalized solvent control) and $2.5 \mathrm{~mL}$ fresh DMEM was applied to $6^{\text {th }}$ Petri dish (medium control). The area around the artificial scratch wound was observed under a light microscope (and their snapshots taken) immediately after adding the solutions and after 16 and 24 hours while incubating the Petri dishes in a humidified atmosphere with $5 \% \mathrm{CO}_{2}$ at $37{ }^{\circ} \mathrm{C}$.

\subsection{Structure-based molecular docking analysis}

To address the molecular mechanisms involved in the antiproliferation effect of T. cilicicus on the A549 cell line, gene expression profiles derived from microarray results of A549 cells grown in ex vivo (4D) and matrigel (3D) models reported by Mishra et al. (2014) was evaluated and the common genes upregulated in both the models was collected (Mishra et al. 2014). The sequences for 9 common genes, namely, EIF5 (Eukaryotic translation initiation factor 5), ABCC8 (ATP-binding cassette sub-family C member 8), GRIK2 (Glutamate receptor ionotropic, kainate 2), COL4A6 (Collagen alpha-6 chain), EPHA7 (Ephrin type-A receptor 7), KIAA1841 (uncharacterized protein), GALNT5 (Polypeptide Nacetylgalactosaminyltransferase 5), DOCK4 (Dedicator of cytokinesis protein 4), and MED31 (Mediator of RNA polymerase II transcription subunit 31) were retrieved from UniProt and their 3D structures were modeled via DeepMind's AlphaFold (The UniProt Consortium 2017; Jumper et al. 2021). A chemical library containing 48 compounds present in the essential oils of T. cilicicus was prepared based on the GC and GC/MS study reported by Tümen et al. (1994) (Tümen et al. 1994). Structure-based molecular docking was performed with each of the 48 ligands against each of 9 protein structures via AutoDock Vina (v1.2.0) using explicit hydrogens, search exhaustiveness of 64, Gasteiger empirical atomic partial charge model, and continuum solvation models following the blind docking protocol (Trott and Olson 2010). Each proteinligand complex was evaluated based on their affinity scores (a scoring function based on binding affinity implemented in AutoDock Vina) and the interaction profile of the top ligand for each protein was visualized via Protein-Ligand Interaction Profiler (PLIP v2.2.2) (Salentin et al. 2015).

\section{Result}

\subsection{Scratch wound assay}

The dry weight of the extracts obtained was measured as $0.201 \mathrm{~g}$, resulting in a yield of $\approx 30.78 \%$. Assessment of $T$. cilicicus wound healing properties was based on scratch wound assay, snapshots taken after the initial application of the extract to the NIH-3T3 coated Petri dishes $(t=0)$, and after 16 hours $(t=16)$, and 24 hours $(\mathrm{t}=24)$ of incubation is shown in Figure 1, the wound healing activity was evaluated based on 
the migration rate of the NIH-3T3 cells over time towards the artificial wound (i.e. healing of the wound) at each concentration compared to the DMSO (solvent control) and DMEM (medium control) Petri dishes at the same time period. As shown in Figure 1, at any timestamp and across each concentration, the migration rate of the NIH-3T3 cells in the medium and DMSO control Petri dishes are higher than the migration rate in the Petri dishes treated with the plant extract, indicating a poor wound healing property (or a strong cytotoxicity). At the 24 hour timestamp, the artificial wound in the medium control is almost recovered, and the artificial wound in the DMSO control is significantly shrunken whereas the opposite is true for the Petri dishes treated with the plant extract.
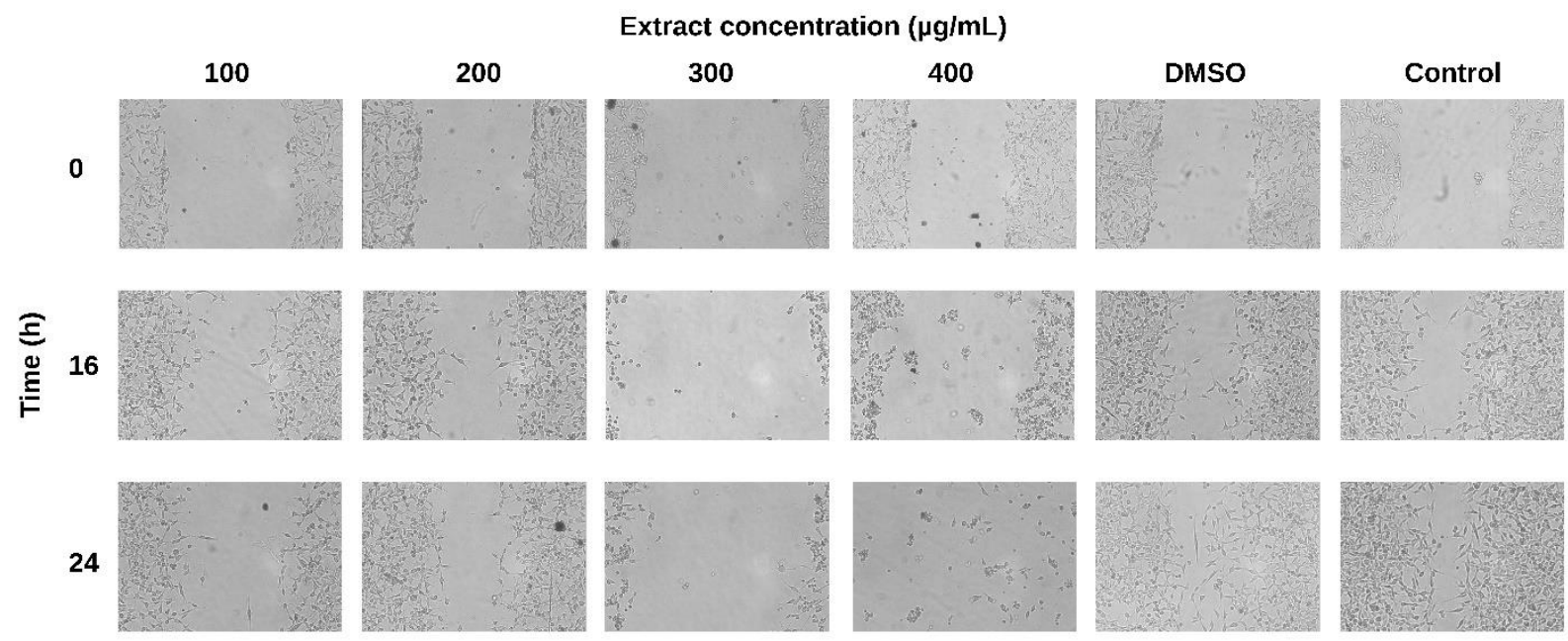

Figure 1. Assessment of T. cilicicus Boiss. \& Bal. wound healing properties. Snapshots were taken immediately after introducing the artificial wound $(\mathrm{t}=0)$, after 16 hours $(\mathrm{t}=16)$, and after 24 hours $(\mathrm{t}=24)$, the extract concentration investigated were $100,200,300$, and $400 \mu \mathrm{g} / \mathrm{mL}$, DMSO Petri contains pure DMSO with DMEM but no plant extract (solvent control) whereas the Control Petri contains no DMSO or extract (medium control).

\subsection{Colorimetric MTT assay}

The dose-response plots (concentration against inhibition percentage) for each cell line investigated via MTT assay are shown in Figure 2, the results are also summarized in Table 1 . By substituting $y=50$ in the line equations (shown on each plot in Figure 2), the $\mathrm{IC}_{50}$ for the NIH-3T3, A549, MDA-MB-231, and DU-145 cell lines was calculated as 556.58, 426.01, 1133.46, and 2219.09 $\mu \mathrm{g} / \mathrm{mL}$, respectively. As the NIH-3T3 cell line is a healthy (not a cancerous) cell line, the relative antiproliferative activity against A549, MDA-MB-231, and DU-145 cell lines were 0.77, 2.04, and 3.99 folds compared to the NIH-3T3 (calculated by dividing cancer cell's $\mathrm{IC}_{50}$ with $\mathrm{NIH}-3 \mathrm{~T} 3$ cell line's $\mathrm{IC}_{50}$ ). This results indicates $T$. cilicucus exerts selective antiproliferative activity on the A549 cell line. It's worth noting that the $\mathrm{R}^{2}$ for the A549 doseresponse plot was calculated as 0.928 whereas for MDA-MB-231 and DU-145 plots it was determined as 0.443 and 0.773 respectively (Figure 2), indicating a higher bias in the calculated $\mathrm{IC}_{50}$ values for the latter 2342 cell lines. 

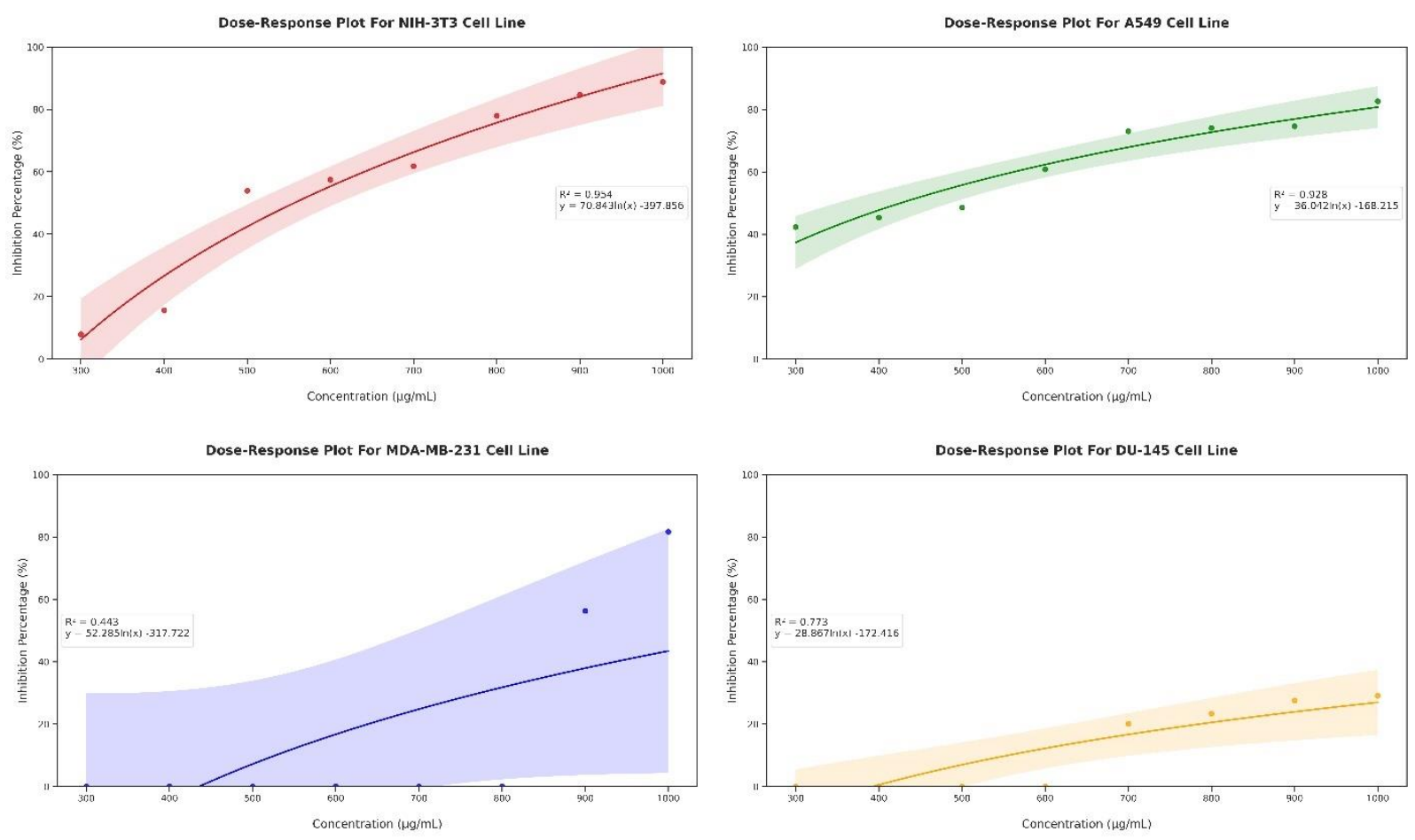

236 Figure 2. Dose-response plots for NIH-3T3, A549, MDA-MB-231, and DU-145 cell lines. Concentration 237 percentages were calculated based on Eq.1 after 24-hour incubation with T. cilicicus extracts.

238 Table 1. Results from MTT assay for NIH-3T3, A549, MDA-MB-231, and DU-145 cell lines 24 hours post-treatment 239 with $T$. cilicicus' extracts.

\begin{tabular}{|c|c|c|c|c|c|c|c|c|}
\hline $\begin{array}{l}\text { Concentration } \\
(\mu \mathrm{g} / \mathrm{mL})\end{array}$ & 1000 & 900 & 800 & 700 & 600 & 500 & 400 & 300 \\
\hline \multicolumn{9}{|c|}{ NIH-3T3 } \\
\hline Cell viability (\%) & $11.19 \pm 0.90$ & $15.36 \pm 0.22$ & $22.06 \pm 2.25$ & $38.20 \pm 5.09$ & $42.55 \pm 2.13$ & $46.10 \pm 6.00$ & $84.4 \pm 12.83$ & $92.0 \pm 27.25$ \\
\hline Inhibition (\%) & 88.81 & 84.64 & 77.94 & 61.8 & 57.45 & 53.90 & 15.59 & 7.93 \\
\hline IC50 & \multicolumn{8}{|c|}{$556.58 \mu \mathrm{g} / \mathrm{mL}$} \\
\hline \multicolumn{9}{|c|}{ A549 } \\
\hline Cell viability (\%) & $17.37 \pm 0.63$ & $25.37 \pm 0.88$ & $25.94 \pm 0.63$ & $26.94 \pm 1.14$ & $39.19 \pm 5.23$ & $51.44 \pm 5.06$ & $54.6 \pm 8.46$ & $57.65 \pm 6.29$ \\
\hline Inhibition (\%) & 82.63 & 74.63 & 74.06 & 73.06 & 60.81 & 48.56 & 45.40 & 42.35 \\
\hline IC50 & $426.01 \mu \mathrm{g} / \mathrm{n}$ & & & & & & & \\
\hline \multicolumn{9}{|c|}{ MDA-MD-231 } \\
\hline Cell viability (\%) & $18.32 \pm 5.70$ & $43.69 \pm 12.60$ & $134.91 \pm 4.03$ & $138.19 \pm 0.44$ & $110.35 \pm 2.55$ & $109.95 \pm 0.65$ & $114.56 \pm 12.2$ & $100.66 \pm 6.02$ \\
\hline Inhibition $(\%)^{* \mathrm{~L}}$ & 81.68 & 56.31 & 0 & 0 & 0 & 0 & 0 & 0 \\
\hline IC50 & \multicolumn{8}{|c|}{$<1133.46 \mu \mathrm{g} / \mathrm{mL}$} \\
\hline \multicolumn{9}{|c|}{ DU-145 } \\
\hline Cell viability (\%) & $70.91 \pm 7.55$ & $72.42 \pm 1.31$ & $76.60 \pm 7.82$ & $79.90 \pm 12.05$ & $140.29 \pm 40.5$ & $115.45 \pm 20.8$ & $116.36 \pm 45.2$ & $100.76 \pm 2.15$ \\
\hline
\end{tabular}


* inhibitions percentages below $0 \%$ (i.e., no inhibition compared to their respective controls) were capped at $0 \%$.

\pm indicates standard deviation.

\& Calculated based on the equations derived in Figure 2 by solving for $\mathrm{y}=50$.

$\mathrm{L}$ The pattern of narrow $\mathrm{IC}_{50}$ range between $800-1000 \mu \mathrm{g} / \mathrm{mL}$ for MDA-MB-231 cell line persisted upon 3 independent repetitions of the experiment.

\section{$241 \quad 3.4$. Structure-based molecular docking}

242

243

244

245

246

The affinities calculated from docking the 9 upregulated proteins in A549 cell lines against the 48 components of $T$. cilicicus essential oils are summarized in Table 2. The raw results from the docking experiment including the structure files are provided in the Supplementary Data 1 (S1).

Table 2. Results for structure-based molecular docking of 48 compounds found in T. cilicicus essential oils against EIF5, ABCC8, GRIK2, COLAA6, EPHA7, KIAA1841, GALNT5, DOCK4, and MED31 proteins.

\begin{tabular}{|c|c|c|c|c|c|c|c|c|c|}
\hline Compound names & EIF5 & ABCC 8 & MED31 & EPHA7 & GALNT5 & COL4A6 & $\begin{array}{r}\text { KIAA184 } \\
1 \\
\end{array}$ & GRIK2 & DOCK4 \\
\hline octanol & -3.70 & -4.50 & -3.80 & -4.40 & -5.10 & -4.30 & -3.90 & -4.50 & -4.50 \\
\hline camphor & -5.20 & -6.00 & -4.40 & -5.00 & -5.30 & -5.00 & -5.80 & -5.40 & -5.80 \\
\hline 1-8-cineole & -4.90 & -6.10 & -4.50 & -4.90 & -5.30 & -5.00 & -5.50 & -5.50 & -6.00 \\
\hline eugenol & -5.10 & -5.90 & -4.70 & -5.40 & -6.80 & -5.10 & -5.60 & -5.90 & -5.90 \\
\hline bornyl acetate & -5.30 & -6.80 & -4.70 & -5.30 & -5.90 & -5.80 & -6.50 & -5.90 & -6.30 \\
\hline $\begin{array}{l}\text { 3-5-5-trimethyl-2- } \\
\text { cyclohexenone }\end{array}$ & -4.90 & -6.00 & -4.70 & -5.00 & -6.10 & -5.20 & -5.30 & -5.20 & -6.10 \\
\hline linalool & -4.40 & -5.50 & -4.70 & -4.50 & -5.40 & -4.90 & -5.00 & -5.00 & -5.40 \\
\hline camphene & -5.00 & -5.80 & -4.50 & -5.00 & -5.60 & -5.50 & -5.30 & -5.30 & -5.90 \\
\hline$\alpha$-pinene & -5.00 & -6.00 & -4.50 & -5.20 & -5.40 & -5.10 & -5.40 & -5.70 & -5.80 \\
\hline carvone & -5.50 & -6.50 & -4.90 & -5.50 & -6.80 & -5.40 & -5.90 & -6.10 & 0.00 \\
\hline$\alpha$-phellandrene & -5.40 & -6.40 & -4.90 & -5.40 & -6.60 & -5.20 & -5.40 & -6.10 & 0.00 \\
\hline$\gamma$-terpinene & -5.00 & -6.40 & -4.70 & -5.50 & -6.80 & -5.10 & -5.40 & -5.90 & -5.90 \\
\hline$\alpha$-terpinene & -5.20 & -6.30 & -5.10 & -5.30 & -6.80 & -5.10 & -5.30 & -5.60 & -6.00 \\
\hline p-cymene & -5.20 & -6.20 & -5.10 & -5.30 & -6.80 & -5.20 & -5.30 & -6.30 & -6.10 \\
\hline $\begin{array}{l}\text { 5-methyl-3- } \\
\text { heptanone }\end{array}$ & -4.00 & -4.60 & -4.10 & -4.30 & -5.30 & -4.10 & -4.30 & -5.20 & -4.80 \\
\hline carvacrol & -5.40 & -6.40 & -5.40 & -5.60 & -7.00 & -5.50 & -5.90 & -6.30 & -6.20 \\
\hline myrtenol & -5.10 & -5.80 & -4.70 & -4.90 & -5.50 & -5.50 & -5.80 & -5.20 & -6.20 \\
\hline terpinen-4-ol & -5.20 & -6.30 & -5.00 & -5.40 & -5.70 & -5.50 & -5.50 & -5.40 & -6.10 \\
\hline terpinolene & -5.20 & -6.70 & -5.30 & -5.40 & -7.20 & -5.30 & -5.40 & -6.00 & -6.10 \\
\hline docosane & -4.40 & -5.70 & -4.40 & -3.90 & -5.30 & -4.40 & -4.50 & -5.40 & -4.80 \\
\hline p-cymen-8-ol & -5.40 & -6.20 & -5.00 & -5.60 & -6.90 & -5.60 & -5.80 & -5.90 & -6.40 \\
\hline$\beta$-pinene & -5.10 & -6.00 & -4.50 & -4.90 & -5.30 & -5.10 & -5.50 & -5.60 & -5.90 \\
\hline$\alpha$-terpineol & -5.10 & -6.10 & -4.80 & -5.40 & -5.70 & -5.40 & -5.40 & -5.70 & -6.00 \\
\hline sabinene & -4.80 & -6.30 & -4.80 & -5.20 & -6.30 & -5.20 & -5.70 & -5.90 & -6.10 \\
\hline 1-octen-3-ol & -3.70 & -4.60 & -4.10 & -4.40 & -5.20 & -4.00 & -4.00 & -4.90 & -4.60 \\
\hline limonene & -4.90 & -6.30 & -5.10 & -5.30 & -6.70 & -5.20 & -5.40 & -6.00 & -5.90 \\
\hline
\end{tabular}




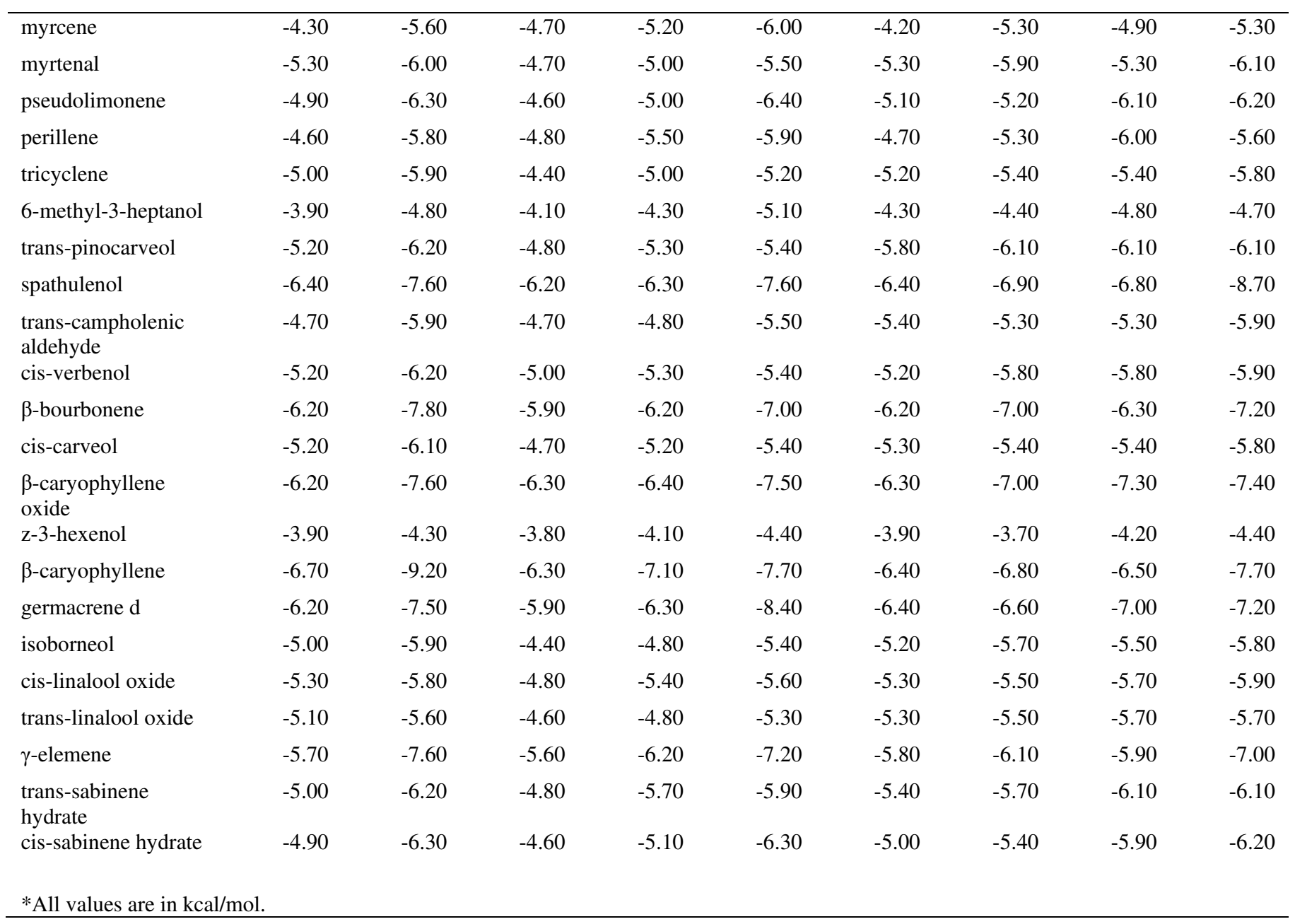

The ligands with the highest affinities for each of the proteins are further visualized in Figure 3. The highest affinities for each protein were EIF5 with $\beta$-caryophyllene $(-6.70 \mathrm{kcal} / \mathrm{mol})$, KIAAI841 with $\beta$-bourbonene and B-caryophyllene oxide $(-7.00 \mathrm{kcal} / \mathrm{mol})$, GALNT5 with Germacrene D $(-8.40 \mathrm{kcal} / \mathrm{mol})$, DOCK4 with Spathulenol (-8.70 kcal/mol), MED31 with $\beta$-bourbonene and B-caryophyllene oxide (-6.30

$252 \mathrm{kcal} / \mathrm{mol}), A B C C 8$ with $\beta$-caryophyllene $(-9.20 \mathrm{kcal} / \mathrm{mol}), C O L A A 6$ with Spathulenol, $\beta$-caryophyllene, and 253 Germacrene D (-6.40 kcal/mol), GRIK2 with B-caryophyllene oxide $(-7.30 \mathrm{kcal} / \mathrm{mol})$, and EPHA7 with $\beta$ 254 caryophyllene $(-7.10 \mathrm{kcal} / \mathrm{mol})$. Both $\beta$-caryophyllene and $\beta$-caryophyllene oxide show strong affinities 255 toward several proteins overexpressed in A549, highlighting their potential role in causing the selective 256 inhibition of the A549 proliferation. 


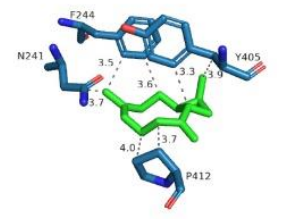

EIF5 - $\beta$-caryophyllene

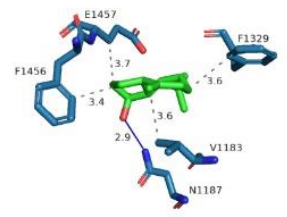

DOCK4 - Spathulenol

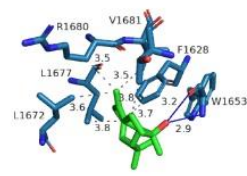

COL4A6 - Spathulenol

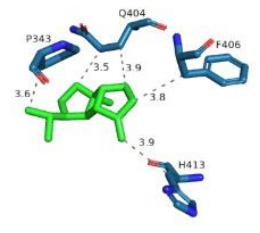

KIAA1841 - $\beta$-bourbonene

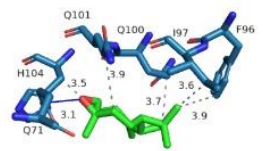

MED31 - $\beta$-caryophyllene oxide

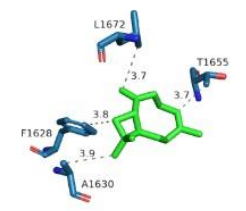

COL4A6 - $\beta$-caryophyllene

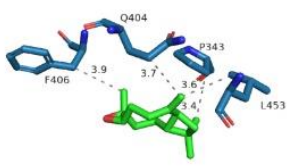

KIAA1841 - $\beta$-caryophyllene oxide

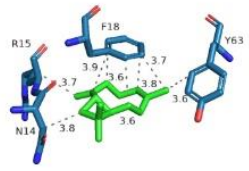

MED31 - $\beta$-caryophyllene

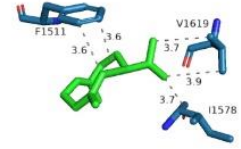

COL4A6 - Germacrene D

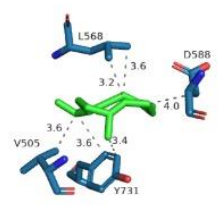

GALNT5 - Germacrene D

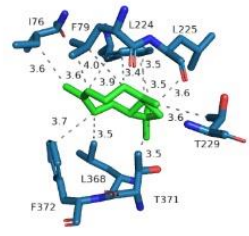

ABCC8 - $\beta$-caryophyllene

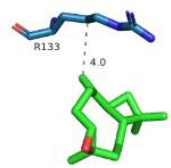

GRIK2 - $\beta$-caryophyllene oxide

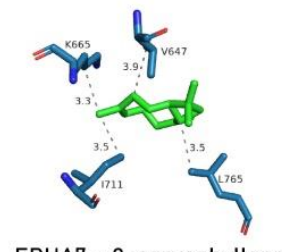

EPHA7 - $\beta$-caryophyllene

Figure 3. Interaction profiles of T. cilicicus essential oil extracts with the highest affinities towards EIF5, ABCC8, GRIK2, COLAA6, EPHA7, KIAA1841, GALNT5, DOCK4, and MED31 proteins. Ligands are shown as green sticks, interacting residues of each protein is shown as blue sticks, hydrophobic interactions are shown in grey dashes, and hydrogen bonds are shown in continuous dark blue lines. All measurements are in $\AA$.

\section{Discussion}

Cancer alone claims millions of lives annually despite the modern therapies and clinical interventions, the sophisticated molecular mechanism and the enormous variation in the disease's genetics and pathology from patient to patient is among the main reasons why a single cure approach is far from achievable, moreover, therapies that work efficiently in certain patients tend to stop working after some time as the development of drug resistance is common among cancer patients (Gottesman et al. 2016; WHO 2021). Therefore, the research for novel anticancer therapeutics remains an intense area of research despite the availability of several chemotherapy agents since the first introduction of nitrogen mustards and antifolate drugs in chemotherapy regimens back in the 1940s (Chabner and Roberts 2005).

This study focused on the traditional method of investigating natural endemic plants used in folk medicine for their potent medicinal property, while several members of the thymus family are known for 
their different medicinal properties, $T$. cilicicus has only been investigated for its antimicrobial activity (Sarac and Ugur 2008). Initially, the ethanolic extract of the T. cilicicus was investigated for its wound healing properties on the NIH-3T3 cell line which yielded poor results as it inhibited the migration of cells even at the $100 \mu \mathrm{g} / \mathrm{mL}$ dose (Figure 1). This result indicated that $T$. cilicicus could hold a strong cytotoxicity effect even at lower doses which was confirmed by colorimetric antiproliferation assays where T. cilicicus extracts inhibited 50\% of the NIH-3T3 cell proliferation at a concentration of $556.58 \mu \mathrm{g} / \mathrm{mL}$ (Figure 2 and Table 1). Colorimetric MTT assay performed on A549, MDA-MB-231, and DU-145 cancer cells following their exposure to T. cilicicus extract for 24 hours has shown some promising results, $T$. cilicicus extract exhibited the weakest inhibitory activity on the DU-145 cell line with an $\mathrm{IC}_{50}$ of $2219.09 \mu \mathrm{g} / \mathrm{mL}$ (4-folds higher than that exhibited on the non-cancerous NIH-3T3 cell line), followed by MDA-MB-231 cell line with an $\mathrm{IC}_{50}$ of $1133.46 \mu \mathrm{g} / \mathrm{mL}$ (Table 2), however, the results from MDA-MB-231 cell line was inconclusive as a pattern of no inhibition was observed at below $800 \mu \mathrm{g} / \mathrm{mL}$ even after 3 independent repetitions of the same experiment, nevertheless, in 3 independent replicas of the experiment, the average $\mathrm{IC}_{50}$ was calculated to be $\approx 950 \mu \mathrm{g} / \mathrm{mL}$, which is still 1.7 -folds higher (but still significant) than that of the non-cancerous NIH-3T3 cell line. The most promising result was achieved on the A549 for which the $\mathrm{IC}_{50}$ was calculated as $426.01 \mu \mathrm{g} / \mathrm{mL}$, lower than the $\mathrm{IC}_{50}$ calculated on the non-cancerous NIH-3T3 cell line, elucidating into a tumor cell-specific antiproliferative activity.

To further investigate the molecular mechanism involved in the selective inhibition of A549 cell growth by T. cilicicus extracts, 9 proteins known for their upregulation in the A549 cell line (both in 3D and $4 \mathrm{D}$ cell cultures) were modeled via AlphaFold from their respective sequences and molecular docking was performed with them against 48 compounds found in the essential oils of T. cilicicus (Mishra et al. 2014; Tümen et al. 1994; Jumper et al. 2021). $\beta$-caryophyllene (which constitutes $1.37 \%$ of the essential oils) achieved the highest affinity against EIF5 (-6.7 kcal/mol), ABCC8 (-9.2 kcal/mol), COL4A6 (-6.4 $\mathrm{kcal} / \mathrm{mol})$, EPHA7 (-7.1 kcal $/ \mathrm{mol})$, and MED31 (-6.3 kcal/mol). $\beta$-caryophyllene oxide (which constitutes $2.53 \%$ of the essential oils) achieved the highest affinity against GRIK2 (-7.3 kcal $/ \mathrm{mol})$, KIAAI841 (-7.0 $\mathrm{kcal} / \mathrm{mol}$ ), and MED31 (-6.3 kcal/mol). Germacrene D (which constitutes $1.08 \%$ of the essential oils) exhibited a high affinity towards COLAA6 $(-6.4 \mathrm{kcal} / \mathrm{mol})$ and $G A L N T 5(-8.4 \mathrm{kcal} / \mathrm{mol})$. Spathulenol (which constitutes $0.29 \%$ of the essential oils) exhibited a high affinity towards DOCK4 $(-8.7 \mathrm{kcal} / \mathrm{mol})$ and $\beta$ bourbonene (which constitutes $0.32 \%$ of the essential oils) exhibited a high affinity towards KIAA1841 ($7.0 \mathrm{kcal} / \mathrm{mol})$.

Downregulation of $M E D 31$ has been reported to suppress the proliferation of osteosarcoma cells and in vitro activation of EPHAl has been shown to promote angiogenesis and tumor growth in hepatocellular carcinoma, the calculated affinity of $\beta$-caryophyllene on the former proteins indicate the potential of a similar antiproliferation mechanism is involved in tumor suppression on the A549 cell line (Buckens et al. 2020; Jiang et al. 2014). GRIK2 has been used as an epigenetic target in gastric cancer for its tumor suppressor role, this could hint into the role of $\beta$-caryophyllene oxide as a GRIK2 agonist in the A549 cell line to inhibit its proliferation (C.-S. Wu et al. 2010). GALNT5 has been reported to be remarkedly upregulated in gastric carcinoma, and in vivo hamsters with knocked-down GALNT5 were reported for low proliferation, migration, and invasion of cholangiocarcinoma cells, Germacrene D affinity towards GALNT5 in the docking study could result in its downregulation in A549 cell line and synergistically exhibit antiproliferative effect along with $\beta$-caryophyllene and $\beta$-caryophyllene oxide (Detarya et al. 2020). DOCK 4 is known to mediate cancer cell migration through activation of RAC1 (by interacting with SH3YL1) in MDA-MB-231 breast cancer and spathulenol's affinity towards it provides some insights into the potency of utilizing spathulenol to block cancer metastasis in in vivo models, moreover, DOCK4mediated $R A C 1$ activation is also involved in MDA-MB-231 breast cancer cell migration, which could explain the uncanonical antiproliferative activity of $T$. cilicicus extracts in the MDA-MB-231 cell line only 
at high concentration (as could be derived from the MDA-MB-231 dose-response plot in Figure 2) as the amount of spathulenol in T. cilicicus constitutes only $0.29 \%$ of its essential oils (Kobayashi et al. 2014).

This study has investigated the medicinal use of endemic T. cilicicus, the antiproliferation assays

\section{Supplementary Materials}

S1: Structure files and raw results from molecular docking.

\section{Competing interests}

The authors declare no competing interests.

\section{Acknowledgment}

The authors are grateful to TUBITAK ULAKBIM, High Performance and Grid Computing Center (TRUBA resources) for the computational resources they had provided for this study.

\section{References}

Abaza, Mohamed Salah I, Khaled Y Orabi, Ebtehal Al-Quattan, and Raja'a J Al-Attiyah. 2015. "Growth Inhibitory and Chemo-Sensitization Effects of Naringenin, a Natural Flavanone Purified from Thymus Vulgaris, on Human Breast and Colorectal Cancer." Cancer Cell International 15 (1): 46. https://doi.org/10.1186/s12935-015-0194-0.

Alarcón, Rocío, Manuel Pardo-de-Santayana, Caroline Priestley, Ramón Morales, and Michael Heinrich. 2015. "Medicinal and Local Food Plants in the South of Alava (Basque Country, Spain)." Journal of Ethnopharmacology 176: 207-24. https://doi.org/10.1016/j.jep.2015.10.022.

Al-Nemari, Rawan, Abdulrahman Al-Senaidy, Abdelhabib Semlali, Mohammad Ismael, Ahmed Yacine Badjah-Hadj-Ahmed, and Abir Ben Bacha. 2020. "GC-MS Profiling and Assessment of Antioxidant, Antibacterial, and Anticancer Properties of Extracts of Annona Squamosa L. Leaves." BMC Complementary Medicine and Therapies 20 (1): 296. https://doi.org/10.1186/s12906-020-03029-9. 
Baser, K Hüsnü Can. 2002. "Aromatic Biodiversity among the Flowering Plant Taxa of Turkey." Pure and Applied Chemistry 74 (4): 527-45. https://doi.org/10.1351/pac200274040527.

Bomfim, Larissa M, Leociley R A Menezes, Ana Carolina B C Rodrigues, Rosane B Dias, Clarissa A Gurgel Rocha, Milena B P Soares, Albertino F S Neto, et al. 2016. "Antitumour Activity of the Microencapsulation of Annona Vepretorum Essential Oil." Basic \& Clinical Pharmacology \& Toxicology 118 (3): 208-13. https://doi.org/https://doi.org/10.1111/bcpt.12488.

Buckens, Oscar J, Btissame El Hassouni, Elisa Giovannetti, and Godefridus J Peters. 2020. "The Role of Eph Receptors in Cancer and How to Target Them: Novel Approaches in Cancer Treatment." Expert Opinion on Investigational Drugs 29 (6): 567-82. https://doi.org/10.1080/13543784.2020.1762566.

Casiglia, Simona, Maurizio Bruno, Massimo Bramucci, Luana Quassinti, Giulio Lupidi, Dennis Fiorini, and Filippo Maggi. 2017. “Kundmannia Sicula (L.) DC: A Rich Source of Germacrene D." Journal of Essential Oil Research 29 (6): 437-42. https://doi.org/10.1080/10412905.2017.1338625.

Chabner, Bruce A, and Thomas G Roberts. 2005. "Chemotherapy and the War on Cancer." Nature Reviews Cancer 5 (1): 65-72. https://doi.org/10.1038/nrc1529.

Chatterjee, Nimrat, and Graham C Walker. 2017. "Mechanisms of DNA Damage, Repair, and Mutagenesis." Environmental and Molecular Mutagenesis 58 (5): 235-63. https://doi.org/10.1002/em.22087.

Cornara, L, A La Rocca, S Marsili, and M G Mariotti. 2009. "Traditional Uses of Plants in the Eastern Riviera (Liguria, Italy)." Journal of Ethnopharmacology 125 (1): 16-30. https://doi.org/10.1016/j.jep.2009.06.021.

Deb, Dipanwita Dutta, G Parimala, S Saravana Devi, and Tapan Chakraborty. 2011. "Effect of Thymol on Peripheral Blood Mononuclear Cell PBMC and Acute Promyelotic Cancer Cell Line HL-60." ChemicoBiological Interactions 193 (1): 97-106. https://doi.org/10.1016/j.cbi.2011.05.009.

Detarya, Marutpong, Kanlayanee Sawanyawisuth, Chaiwat Aphivatanasiri, Sriwipa Chuangchaiya, Paksiree Saranaruk, Lukkana Sukprasert, Atit Silsirivanit, Norie Araki, Sopit Wongkham, and Chaisiri Wongkham. 2020. "The O-GalNAcylating Enzyme GALNT5 Mediates Carcinogenesis and Progression of Cholangiocarcinoma via Activation of AKT/ERK Signaling." Glycobiology 30 (5): 31224. https://doi.org/10.1093/glycob/cwz098.

Doosti, Mohammad-Hossein, Kazem Ahmadi, and Mahdi Fasihi-Ramandi. 2018. "The Effect of Ethanolic Extract of Thymus Kotschyanus on Cancer Cell Growth in Vitro and Depression-like Behavior in the Mouse." Journal of Traditional and Complementary Medicine 8 (1): 89-94. https://doi.org/https://doi.org/10.1016/j.jtcme.2017.03.003.

Elbe, Hulya, Gurkan Yigitturk, Turker Cavusoglu, Tuba Baygar, Melike Ozgul Onal, and Feral Ozturk. 2020. "Comparison of Ultrastructural Changes and the Anticarcinogenic Effects of Thymol and Carvacrol on Ovarian Cancer Cells: Which Is More Effective?" Ultrastructural Pathology 44 (2): 193-202. https://doi.org/10.1080/01913123.2020.1740366. 
Erenler, Ramazan, Ozkan Sen, Ilyas Yildiz, and Ali Aydın. 2016. "Antiproliferative Activities of Chemical Constituents Isolated from Thymus Praecox Subsp. Grossheimii (Ronniger) Jalas." Records of Natural Products, no. 6 November-December: 766-70.

Esmaeili-Mahani, Saeed, Farzaneh Falahi, and Mohammad Mehdi Yaghoobi. 2014. "Proapoptotic and Antiproliferative Effects of Thymus Caramanicus on Human Breast Cancer Cell Line (MCF-7) and Its Interaction with Anticancer Drug Vincristine." Edited by Mohd Roslan Sulaiman. Evidence-Based Complementary and Alternative Medicine 2014: 893247. https://doi.org/10.1155/2014/893247.

Everest, Ayse, and Ersin Ozturk. 2005. "Focusing on the Ethnobotanical Uses of Plants in Mersin and Adana Provinces (Turkey)." Journal of Ethnobiology and Ethnomedicine 1 (1): 6. https://doi.org/10.1186/1746-4269-1-6.

Fidyt, Klaudyna, Anna Fiedorowicz, Leon Strządała, and Antoni Szumny. 2016. " $\beta$-Caryophyllene and $\beta$ Caryophyllene Oxide-Natural Compounds of Anticancer and Analgesic Properties." Cancer Medicine 5 (10): 3007-17. https://doi.org/10.1002/cam4.816.

GIACOMO, SILVIA DI, ANTONELLA DI SOTTO, GABRIELA MAZZANTI, and MICHAEL WINK. 2017. "Chemosensitizing Properties of $\beta$-Caryophyllene and $\beta$-Caryophyllene Oxide in Combination with Doxorubicin in Human Cancer Cells." Anticancer Research 37 (3): 1191 LP - 1196.

Gordo, Joana, Patrícia Máximo, Eurico Cabrita, Ana Lourenço, Abel Oliva, Joana Almeida, Mariana Filipe, et al. 2012. "Thymus Mastichina: Chemical Constituents and Their Anti-Cancer Activity." Natural Product Communications 7 (11): 1934578X1200701120. https://doi.org/10.1177/1934578X1200701120.

Gottesman, Michael M, Orit Lavi, Matthew D Hall, and Jean-Pierre Gillet. 2016. "Toward a Better Understanding of the Complexity of Cancer Drug Resistance." Annual Review of Pharmacology and Toxicology 56: 85-102. https://doi.org/10.1146/annurev-pharmtox-010715-103111.

Gürdal, Bahar, and Sükran Kültür. 2013. "An Ethnobotanical Study of Medicinal Plants in Marmaris (Muğla, Turkey)." Journal of Ethnopharmacology 146 (1): 113-26. https://doi.org/10.1016/j.jep.2012.12.012.

Heidari, Zahra, Ali Salehzadeh, Seyed Ataollah Sadat Shandiz, and Sara Tajdoost. 2018. "Anti-Cancer and Anti-Oxidant Properties of Ethanolic Leaf Extract of Thymus Vulgaris and Its Bio-Functionalized Silver Nanoparticles." 3 Biotech 8 (3): 177. https://doi.org/10.1007/s13205-018-1199-x.

Hunter, J D. 2007. "Matplotlib: A 2D Graphics Environment." Computing in Science I\& Engineering 9 (3): 90-95. https://doi.org/10.1109/MCSE.2007.55.

Imran, Muhammad, Tanweer Aslam Gondal, Muhammad Atif, Muhammad Shahbaz, Tahira Batool Qaisarani, Muhammad Hanif Mughal, Bahare Salehi, Miquel Martorell, and Javad Sharifi-Rad. 2020. "Apigenin as an Anticancer Agent." Phytotherapy Research 34 (8): 1812-28. https://doi.org/https://doi.org/10.1002/ptr.6647.

Jiang, Chaoyin, Hua Chen, Lei Shao, and Qiaojie Wang. 2014. "MicroRNA-1 Functions as a Potential Tumor Suppressor in Osteosarcoma by Targeting Med1 and Med31." Oncol Rep 32 (3): 1249-56. https://doi.org/10.3892/or.2014.3274. 
Jumper, John, Richard Evans, Alexander Pritzel, Tim Green, Michael Figurnov, Olaf Ronneberger, Kathryn Tunyasuvunakool, et al. 2021. "Highly Accurate Protein Structure Prediction with AlphaFold." Nature 596 (7873): 583-89. https://doi.org/10.1038/s41586-021-03819-2.

Kaya, Ergun, Mehmet Ali Balci, Omer Akguller, Selin Galatali, Sevil Yeniocak, Taner Mercan, Sevinc Guldag, et al. 2021. "Development of an Optimum Proliferation Medium via the Graph Kernel Statistical Analysis Method for Genetically Stable in Vitro Propagation of Endemic Thymus Cilicicus (Turkey)." Acta Botanica Croatica 80 (2). https://doi.org/10.37427/botcro-2021-024.

Kobayashi, Masakazu, Kohei Harada, Manabu Negishi, and Hironori Katoh. 2014. "Dock4 Forms a Complex with SH3YL1 and Regulates Cancer Cell Migration." Cellular Signalling 26 (5): 1082-88. https://doi.org/https://doi.org/10.1016/j.cellsig.2014.01.027.

Koparal, A Tansu, and Melih Zeytinoglu. 2003. "Effects of Carvacrol on a Human Non-Small Cell Lung Cancer (NSCLC) Cell Line, A549." Cytotechnology 43 (1-3): 149-54. https://doi.org/10.1023/b:cyto.0000039917.60348.45.

Li, Xiao, Ting He, Xiuhuan Wang, Meng Shen, Xin Yan, Shusheng Fan, Le Wang, et al. 2019. "Traditional Uses, Chemical Constituents and Biological Activities of Plants from the Genus Thymus." Chemistry \& Biodiversity 16 (9): e1900254. https://doi.org/10.1002/cbdv.201900254.

Li, Yi, Jia-ming Wen, Chuan-jun Du, Su-min Hu, Jia-xi Chen, Shi-geng Zhang, Nan Zhang, et al. 2017. "Thymol Inhibits Bladder Cancer Cell Proliferation via Inducing Cell Cycle Arrest and Apoptosis." Biochemical and Biophysical Research Communications 491 (2): 530-36. https://doi.org/10.1016/j.bbrc.2017.04.009.

Llana-Ruiz-Cabello, María, Daniel Gutiérrez-Praena, Silvia Pichardo, F Javier Moreno, José María Bermúdez, Susana Aucejo, and Ana María Cameán. 2014. "Cytotoxicity and Morphological Effects Induced by Carvacrol and Thymol on the Human Cell Line Caco-2." Food and Chemical Toxicology 64: 281-90. https://doi.org/10.1016/j.fct.2013.12.005.

Lodish, Harvey F, Arnold Berk, Chris Kaiser, Monty Krieger, Matthew P Scott, Anthony Bretscher, Hidde L Ploegh, and Paul T Matsudaira. 2008. Molecular Cell Biology. 5th ed. W.H. Freeman.

Martins-Gomes, Carlos, Eliana B Souto, Fernanda Cosme, Fernando M Nunes, and Amélia M Silva. 2019. "Thymus Carnosus Extracts Induce Anti-Proliferative Activity in Caco-2 Cells through Mechanisms That Involve Cell Cycle Arrest and Apoptosis." Journal of Functional Foods 54: 128-35. https://doi.org/10.1016/j.jff.2019.01.010.

Martins-Gomes, Carlos, Meriem Taghouti, Judith Schäfer, Mirko Bunzel, Amélia M Silva, and Fernando M Nunes. 2018. "Chemical Characterization and Bioactive Properties of Decoctions and Hydroethanolic Extracts of Thymus Carnosus Boiss." Journal of Functional Foods 43: 154-64. https://doi.org/https://doi.org/10.1016/j.jff.2018.02.012.

Mishra, Dhruva K, Chad J Creighton, Yiqun Zhang, Don L Gibbons, Jonathan M Kurie, and Min P Kim. 2014. "Gene Expression Profile of A549 Cells from Tissue of 4D Model Predicts Poor Prognosis in Lung Cancer Patients." International Journal of Cancer 134 (4): 789-98. https://doi.org/10.1002/ijc.28428. 
Nabavi, Seyed Mohammad, Anna Marchese, Morteza Izadi, Valeria Curti, Maria Daglia, and Seyed Fazel Nabavi. 2015. "Plants Belonging to the Genus Thymus as Antibacterial Agents: From Farm to Pharmacy." Food Chemistry 173: 339-47. https://doi.org/10.1016/j.foodchem.2014.10.042.

Newman, D J, G M Cragg, and K M Snader. 2000. "The Influence of Natural Products upon Drug Discovery." Natural Product Reports 17 (3): 215-34. https://doi.org/10.1039/a902202c.

Patel, Bhushankumar, Vichiksha R Shah, and Supriya A Bavadekar. 2012. "Anti-Proliferative Effects of Carvacrol on Human Prostate Cancer Cell Line, LNCaP." The FASEB Journal 26 (S1): 1037.5-1037.5. https://doi.org/https://doi.org/10.1096/fasebj.26.1_supplement.1037.5.

QingHua, Yin, Zhuang YinZhi, and Yan FengXiang. 2010. "Antitumor efficacy of thymol." Progress in Modern Biomedicine 10 (11): 2073-75.

Rowshan, Vahid, Atefeh Bahmanzadegan, and Mohammad Jamal Saharkhiz. 2013. "Influence of Storage Conditions on the Essential Oil Composition of Thymus Daenensis Celak." Industrial Crops and Products 49: 97-101. https://doi.org/10.1016/j.indcrop.2013.04.029.

Salehi, Bahare, Mohammad Sanad Abu-Darwish, Amer Hussein Tarawneh, Celia Cabral, Anastassiya V Gadetskaya, Ligia Salgueiro, Tahereh Hosseinabadi, et al. 2019. “Thymus Spp. Plants - Food Applications and Phytopharmacy Properties." Trends in Food Science \& Technology 85: 287-306. https://doi.org/10.1016/j.tifs.2019.01.020.

Salentin, Sebastian, Sven Schreiber, V Joachim Haupt, Melissa F Adasme, and Michael Schroeder. 2015. "PLIP: Fully Automated Protein-Ligand Interaction Profiler." Nucleic Acids Research 43 (W1): W443-47. https://doi.org/10.1093/nar/gkv315.

Sarac, Nurdan, and Aysel Ugur. 2008. "Antimicrobial Activities of the Essential Oils of Origanum Onites L., Origanum Vulgare L. Subspecies Hirtum (Link) letswaart, Satureja Thymbra L., and Thymus Cilicicus Boiss. \& Bal. Growing Wild in Turkey." Journal of Medicinal Food 11 (3): 568-73. https://doi.org/10.1089/jmf.2007.0520.

Schenone, Monica, Vlado Dančík, Bridget K Wagner, and Paul A Clemons. 2013. "Target Identification and Mechanism of Action in Chemical Biology and Drug Discovery." Nature Chemical Biology 9 (4): 232-40. https://doi.org/10.1038/nchembio.1199.

Seresht, Hasan Rezaei, Bushra Jabbar Albadry, Aseel Kamil Mohammed Al-mosawi, Omid Gholami, and Hamid Cheshomi. 2019. "The Cytotoxic Effects of Thymol as the Major Component of Trachyspermum Ammi on Breast Cancer (MCF-7) Cells." Pharmaceutical Chemistry Journal 53 (2): 101-7. https://doi.org/10.1007/s11094-019-01961-w.

Solecki, Ralph S. 1975. "Shanidar IV, a Neanderthal Flower Burial in Northern Iraq." Science 190 (4217): 880 LP - 881. https://doi.org/10.1126/science.190.4217.880.

Sudhakar, Akulapalli. 2009. "History of Cancer, Ancient and Modern Treatment Methods." Journal of Cancer Science \& Therapy 01 (02): i-iv. https://doi.org/10.4172/1948-5956.100000e2.

Sultan, Nahla, Azza I Othman, Mohamed A El-Missiry, Aly Fahmy Mohamed, and Sameh M Shabana. 2019. "Assessment of the Anticancer Activity of Caryophyllene Oxide against Breast Cancer Cell 
Line and Related Genetic Alterations: In Vitro Study." Journal of Environmental Sciences. Mansoura University 48 (2): 87-94. https://doi.org/10.21608/joese.2019.158392.

Sun, Zhen-Xiao, Ying-Hui Zhang, Shuang Cheng, Qing-Wen Ma, Shan-Li Guo, and Jin-Bao Zhang. 2005. "Anti-tumor effect of ethanol extracts from Thymus quinquecostatus Celak on human leukemia cell line." Zhong xi yi jie he xue bao = Journal of Chinese integrative medicine 3 (5): 382-85. https://doi.org/10.3736/jcim20050513.

The UniProt Consortium. 2017. "UniProt: The Universal Protein Knowledgebase." Nucleic Acids Research 45 (D1): D158-69. https://doi.org/10.1093/nar/gkw1099.

Trott, Oleg, and Arthur J Olson. 2010. "AutoDock Vina: Improving the Speed and Accuracy of Docking with a New Scoring Function, Efficient Optimization, and Multithreading." Journal of Computational Chemistry 31 (2): 455-61. https://doi.org/10.1002/jcc.21334.

Tümen, G, M Koyuncu, N Kirimer, and K H C Baser. 1994. "Composition of the Essential Oil of Thymus Cilicicus Boiss. \& Bal." Journal of Essential Oil Research 6 (1): 97-98. https://doi.org/10.1080/10412905.1994.9698336.

Virtanen, Pauli, Ralf Gommers, Travis E Oliphant, Matt Haberland, Tyler Reddy, David Cournapeau, Evgeni Burovski, et al. 2020. "SciPy 1.0: Fundamental Algorithms for Scientific Computing in Python." Nature Methods 17: 261-72. https://doi.org/10.1038/s41592-019-0686-2.

Wang, Zhong, Feng Liu, Jian-Jun Yu, and Ji-Zhong Jin. 2018. " $\beta$-Bourbonene Attenuates Proliferation and Induces Apoptosis of Prostate Cancer Cells." Oncology Letters 16 (4): 4519-25. https://doi.org/10.3892/ol.2018.9183.

Waskom, Michael L. 2021. "Seaborn: Statistical Data Visualization." Journal of Open Source Software 6 (60): 3021. https://doi.org/10.21105/joss.03021.

WHO. 2021. "Cancer." WHO Statistics. 2021. https://www.who.int/news-room/factsheets/detail/cancer.

Wu, Chi-Sheng, Yen-Jung Lu, Hsin-Pai Li, Chuen Hsueh, Chang-Yi Lu, Yu-Wei Leu, Hao-Ping Liu, KwangHuei Lin, Tim Hui-Ming Huang, and Yu-Sun Chang. 2010. "Glutamate Receptor, Ionotropic, Kainate 2 Silencing by DNA Hypermethylation Possesses Tumor Suppressor Function in Gastric Cancer." International Journal of Cancer 126 (11): 2542-52. https://doi.org/10.1002/ijc.24958.

Wu, Shuang, Feng-Xiang Wei, Hong-Zhi Li, Xiao-Guang Liu, Jian-Hua Zhang, and Jun-Xing Liu. 2013. "Chemical composition of essential oil from Thymus citriodorus and its toxic effect on liver cancer cells." Zhong yao cai = Zhongyaocai = Journal of Chinese medicinal materials 36 (5): 756-59.

Yin, Qing-Hua, Feng-Xiang Yan, Xu-Yu Zu, You-Hua Wu, Xiao-Ping Wu, Ming-Chu Liao, Shu-Wen Deng, Lei-Lan Yin, and Ying-Zhi Zhuang. 2012. "Anti-Proliferative and pro-Apoptotic Effect of Carvacrol on Human Hepatocellular Carcinoma Cell Line HepG-2." Cytotechnology 64 (1): 43-51. https://doi.org/10.1007/s10616-011-9389-y.

Zarai, Zied, Adel Kadri, Ines Ben Chobba, Riadh Ben Mansour, Ahmed Bekir, Hafedh Mejdoub, and Néji Gharsallah. 2011. "The In-Vitro Evaluation of Antibacterial, Antifungal and Cytotoxic Properties of 
545

546

547

548

549
Marrubium Vulgare L. Essential Oil Grown in Tunisia." Lipids in Health and Disease 10 (1): 161. https://doi.org/10.1186/1476-511X-10-161.

Zeng, Qiongyao, Yuncheng Che, Yu Zhang, Mei Chen, Qiang Guo, and Wenjing Zhang. 2020. "Thymol Isolated from Thymus Vulgaris L. Inhibits Colorectal Cancer Cell Growth and Metastasis by Suppressing the Wnt/ $\beta$-Catenin Pathway." Drug Design, Development and Therapy 14 (July): 253547. https://doi.org/10.2147/DDDT.S254218. 